\title{
Assessment and Management of Infection in Alcoholic Hepatitis
}

\author{
Nikhil Vergis, MRCP, $\mathrm{PhD}^{1} \quad$ Stephen R. Atkinson, MRCP, $\mathrm{PhD}^{1} \quad$ Mark R. Thursz, FRCP, MD ${ }^{1}$ \\ ${ }^{1}$ Department of Surgery \& Cancer, Division of Digestive Diseases, \\ Address for correspondence Mark R. Thursz, FRCP, MD, Division of \\ Imperial College London, London, United Kingdom \\ Digestive Diseases, Department of Surgery \& Cancer, Imperial College \\ London, London, United Kingdom (e-mail: m.thursz@imperial.ac.uk).
} Semin Liver Dis 2020;40:11-19.

\begin{abstract}
Keywords

- alcoholic hepatitis

- infection

- polymerase chain reaction

- systemic inflammatory response syndrome

Severe alcoholic hepatitis $(\mathrm{SAH})$ is a condition characterized by jaundice and liver failure that develops after heavy and prolonged alcohol consumption. Infection frequently complicates the natural history of the disease and is independently associated with mortality. Objective recognition and recording of infection are therefore essential in the evaluation of therapeutic interventions and for antibiotic stewardship. This review will evaluate infections that complicate SAH at admission and beyond. Factors that associate with the development of infection will be identified and clinical and laboratory techniques available to identify infection will be discussed. Common pathogens and frequently used antibiotics will be reviewed and recommendations will be made for the management of infection for SAH patients. New techniques to assess infection earlier and more precisely may improve diagnosis and treatment of this important driver of mortality in SAH.
\end{abstract}

Alcoholic hepatitis (AH) is an in acute inflammatory condition characterized by jaundice and liver failure that develops after heavy and prolonged alcohol consumption. ${ }^{1}$ It is a unique subgroup within the broader syndrome of acute decompensation of cirrhosis (AD) that includes steatohepatitis on liver biopsy. Patients with Maddrey's discriminant function $\geq 32$ have severe alcoholic hepatitis (SAH) and are candidates for treatment but therapy may involve immunosuppression. ${ }^{2}$

Infection frequently complicates the natural history of the disease and is independently associated with mortality. ${ }^{3}$ Objective recognition and recording of infection are therefore essential in the evaluation of therapeutic interventions. This review will evaluate infections that complicate SAH at admission and nosocomial infections that may be associated with immunosuppressive therapy. Factors associated with the development of infection will be identified. Clinical and laboratory techniques to identify infection will be evaluated. Management of infection in the context of SAH will be discussed.

A central issue in reporting and managing infection is the difficulty in making an accurate diagnosis. This is mainly because the evaluation of both microorganism and host response is complex in the setting of SAH. Microbiological techniques have advanced from identification of viable organism by microbial culture to detection of nucleic acid by polymerase chain reaction; however, differentiating active infection from the intestinal leak of microbial products remains challenging. Similarly, immunological advances have progressed the field from traditional enzyme-linked immunosorbent assay (ELISA)-based detection of secreted serum proteins to cytometric immunophenotyping of the host response at the level of the single cell. However, immune signatures with the necessary specificity for diagnosis of infection to guide antimicrobial stewardship have proved elusive.

Management of suspected infection in hospitalized patients has changed considerably over the past 20 years. The practice of delaying antimicrobial therapy until microbiological evidence confirming infection is obtained has been replaced by an urgency to institute antimicrobial therapy as soon as infection is suspected, with an emphasis on antibiotic therapy within the so-called "golden first hour" advocated by global initiatives such as the Surviving Sepsis campaign. ${ }^{4-6}$ Separately, the capacity for intensive care management of infected patients with acute on chronic liver failure (ACLF) has expanded. ${ }^{7}$ These changes in clinical published online

August 1, 2019
DOI https://doi.org/ 10.1055/s-0039-1693402. ISSN 0272-8087.
Copyright $\odot 2020$ by Thieme Medical Publishers, Inc., 333 Seventh Avenue, New York, NY 10001, USA. Tel: +1(212) 760-0888.
License terms

(1) (1) 
practice confound comparison between studies conducted during this period. Moreover, while overall mortality from ACLF appears to have fallen, ${ }^{7}$ some series report persistently high rates of sepsis in cirrhotic patients despite the surviving sepsis campaign, purportedly secondary to multidrugresistant (MDR) organisms. ${ }^{8}$

\section{SAH Patients Presenting with Infection}

Several studies have defined the natural history of infection for patients with $\mathrm{AH} .{ }^{9}$ Differences in outcomes for infections present at the time of admission and infections arising after initiation of treatment were first identified by Louvet et al. ${ }^{10}$ The largest single study analysis of infection was conducted from patients recruited to the STeroids Or Pentoxyfilline for Alcoholic Hepatitis (STOPAH) trial between 2011 and 2014, in which 1092 patients who met clinical criteria for SAH were randomly allocated into a group of 547 patients treated with 28 days of prednisolone and 545 were not. ${ }^{2}$ In the STOPAH study, infections between admission and start of therapy occurred in $12 \%$ of patients. Data suggest that if these baseline infections are controlled, patients can start on corticosteroid therapy without major implications for prognosis. ${ }^{3,10}$ However, most available literature in SAH derives from analyses that exclude patients in whom baseline infection is not controlled. These infected patients may be classified as $A D$ or ACLF and there are several reports that admission infections triggering ACLF portend poor shortterm prognosis. ${ }^{11}$ In a French study that recruited 246 patients with biopsy proven $\mathrm{SAH}, 5 / 6$ patients in whom baseline infection could not be controlled died from the baseline infection. ${ }^{10}$ Further insight into these infections comes from the STOPAH study, in which the mortality of patients infected at admission and in whom antibiotics were stopped was markedly higher than the mortality for patients with infection at admission for whom antibiotics were continued while prednisolone was initiated. ${ }^{3}$ These data emphasize the dangers of baseline infection for SAH patients if not effectively treated.

\section{Incident Infection}

Infection that develops after initiation of corticosteroid therapy, up to a period of 90 days, is defined as incident infection. Incident infections are often nosocomial, and may be associated with MDR organisms. These infections are also associated with an immune paresis that may be exacerbated by immunosuppressive therapy. Accordingly, the impact of incident infection on mortality was independent of liver function during the STOPAH study in patients treated with prednisolone but was secondary to liver function in patients treated without corticosteroids.

\section{Treatment-Associated Infection}

No trial has demonstrated benefit from corticosteroid therapy at 90 days, in part related to the development of late infections associated with prednisolone therapy that negates early benefit from immunosuppression. . $^{3,12}$
Serious infections and infections occurring between 28 and 90 days increased by approximately 30 and 70\% with prednisolone, respectively. Infection that developed within 7 days of instituting prednisolone decreased the likelihood of a treatment response, assessed using a Lille score $<0.45$. $^{3}$ Patients treated with corticosteroids who become infected have a $30 \%$ increased mortality at 2 months ${ }^{10}$ and twofold at 3 months. $^{2}$

There is an increased incidence of lung infection ${ }^{10}$ during hospital treatment but data from the STOPAH study suggest that prednisolone does not specifically heighten the risk of pneumonia. Gustot et al found invasive aspergillosis infection in $21 \%(15 / 70)$ of patients treated with corticosteroid and $11 \%(2 / 19)$ of patients who had no specific treatment for $\mathrm{SAH}$. These cases of invasive aspergillosis were refractory to standard antifungal therapy and all patients developing the complication died. ${ }^{13}$

Post-hoc analyses of the STOPAH data have identified two populations of patients with SAH that appear to retain benefit from corticosteroid therapy until 90 days. First, low levels of circulating $16 \mathrm{~S}$ bacterial deoxyribonucleic acid (DNA) at baseline indicate a lower risk of developing infection within the first 7 days. With holding prednisolone from patients with high circulating bacterial DNA levels may reduce infectious complications from prednisolone and was modeled to result in a survival benefit for prednisolone by 90 days. $^{3}$ Similarly, withholding prednisolone from patients with high neutrophil to lymphocyte ratio was demonstrated to result in survival benefit for prednisolone at 90 days. $^{14}$

Baseline static scores of liver function have been considered to stratify patients for prednisolone therapy. Patients with SAH and model for end-stage liver disease (MELD) $<25$ represent a subgroup of patients with lower risk of mortality (9\%) in whom there may be reduced benefit from corticosteroid therapy. ${ }^{15}$ On the other hand, patients with high Age Bilirubin INR Creatinine (ABIC) or Glasgow Alcoholic Hepatitis Score (GAHS) above 6.71 and 8 , respectively, showed a survival benefit from steroids in subgroup analysis at 28 days. ${ }^{15}$

The impact of organ failure on the risk of infection and utility of prednisolone has been assessed. Higher stages of ACLF were associated with higher rates of infection and a reduced possibility of a response to corticosteroid therapy. ${ }^{16}$ However, if Lille response could be demonstrated there were improved survival prospects for patients irrespective of the grade of ACLF in the STOPAH cohort. Independent cohorts of 165 and 97 patients with SAH confirmed that infection is strongly associated with the subsequent development of organ failure (ACLF) in these patients. ${ }^{17}$ Incident infections in these cohorts of patients led to a more than 27-fold increased risk of mortality at 28 days on multivariable analysis. ${ }^{17}$

\section{Predictors of Infection}

Immunotherapy is a promising avenue for therapeutic research in SAH. However, there are clear risks to be considered in this group of patients. Early identification of patients at risk of infections may facilitate safer immunotherapy. 
Several studies have examined clinical predictors of infection in SAH patients who develop nosocomial infection. Factors such as age, baseline liver function, improvement in cholestasis within 7 days, renal function, and the systemic inflammatory response syndrome (SIRS) correlate with the likelihood of developing infections after the start of therapy. $3,10,18$ In one study that compared SAH patients with SIRS criteria who did and did not have infection, liver function was a key predictor of the likelihood of developing infection. $^{18}$ More recently, high levels of circulating $16 S$ bacterial DNA have been shown to confer a greater than fourfold increased risk of developing infection if patients are treated with prednisolone, independent of liver function. ${ }^{3}$

\section{Clinical Diagnosis of Infection}

Reported infection rates in SAH studies vary widely from 14 to $30 \%$ at admission and from 8 to $51 \%$ of patients infected after the start of treatment. ${ }^{9}$ There are several reasons for the broad range of reported infection rates: these include variation in prescription of prophylactic antibiotics; global antimicrobial resistance profiles; severity of underlying liver disease; frequency and completeness of infection screening panels; and fundamental differences in the criteria used to diagnose infection.

Assessment for infection in any hospitalized patient requires careful review of symptoms, thorough clinical examination and basic investigations such as urinalysis, blood culture, chest radiography and, when ascites is present, microscopy and culture of ascitic fluid. ${ }^{19}$ Additional samples may also be required according to symptoms, such as sputum for culture and microscopy in the case of suspected purulent respiratory infection. Elevated C-reactive protein (CRP) and white cell count may suggest infection. However, infection may also be suspected in the absence of objective evidence when there are changes in vital signs, rise in bilirubin or deterioration of renal function.

\section{Patients Meeting the North American Consortium for the Study of End-Stage Liver Disease (NACSELD) Criteria for Infection}

Laboratory identification of a culprit pathogen is considered the gold standard for diagnosis of infection. However, an organism was isolated from body fluid culture in only $40 \%$ of infections in the STOPAH study, ${ }^{3}$ emphasizing the need for alternative criteria. Previous studies of infection in SAH have incorporated variable systemic inflammatory criteria for the inclusion of patients in whom a microorganism is not identified. For example, Michelena et al diagnosed and treated infection in patients with an identifiable focus of infection or a temperature above $38^{\circ} \mathrm{C},{ }^{18}$ while Louvet et al included all patients with fever above $38.5^{\circ} \mathrm{C}^{10}$ An instructive report from the NACSELD consortium detailed criteria for infection in patients with chronic liver disease ( $\mathbf{- T a b l e ~} \mathbf{1}$ ).

Common sites for infection and prevalence of pathogens in SAH patients are reviewed in recent literature. ${ }^{9}$ Of note, lung infections and spontaneous bacterial peritonitis are the commonest sites of infection, with lung infections accounting for approximately $40 \%$ of infection ${ }^{3}$ (-Table 2 ). The proportion of infections arising from the lung appears to be higher in SAH compared with patients with AD globally (-Table 2). Escherichia coli is the commonest pathogen that can be isolated from body fluid of SAH patients using standard microbiological culture techniques ${ }^{3}$ (-Table 3 ).

\section{Limitations in the NACSELD Criteria for Infection in SAH Patients}

Isolation of an organism using standard microbiological techniques requires 1 to 2 days: this delay is clearly too long for critically unwell patients with SAH. In addition, a proportion of pathogenic organisms will not grow in standard laboratory media, a situation exacerbated by the widespread use of broad-spectrum antibiotics. Further, false positive culture of bodily fluids can arise from skin contaminants and interpretation of this is challenging if vascular

Table 1 Clinical, microbiological, and radiological criteria defined by the NACSELD consortium for the diagnosis of infection

(i) Spontaneous bacteremia: positive blood cultures without a source of infection, OR

(ii) SBP: ascitic fluid polymorphonuclear cells $>250 / \mu \mathrm{L}$ with/without a positive fluid culture, OR

(iii) Lower respiratory tract infections: new pulmonary infiltrate in the presence of: (a) at least one respiratory symptom (cough, sputum production, dyspnea, pleuritic pain) with (b) at least one finding on auscultation (rales or crepitation) or (c) one sign of infection (core body temperature $>38^{\circ} \mathrm{C}$ or $<36^{\circ} \mathrm{C}$, shivering or leucocyte count $>10,000 / \mathrm{mm}^{3} \mathrm{or}<4,000 / \mathrm{mm}^{3}$ ) in the absence of antibiotics, OR

(iv) Clostridium difficile: diarrhea with a positive $C$. difficile assay

(v) Bacterial enterocolitis: diarrhea or dysentery with a positive stool culture for Salmonella, Shigella, Yersinia, Campylobacter, or pathogenic Escherichia coli, OR

(vi) Skin Infection: fever with cellulitis, OR

(vii) UTI: urine WBC > 15/high power field with either positive urine gram stain or culture in a symptomatic patient, OR

(viii) Intra-abdominal infections: diverticulitis, appendicitis, cholangitis, etc., OR

(ix) Secondary bacterial peritonitis: $>250$ polymorphonuclear cells $/ \mu \mathrm{L}$ of ascitic fluid in the presence of an intra-abdominal source of peritonitis and multiple organisms cultured from ascitic fluid.

Abbreviations: NACSELD, North American Consortium for the Study of End-Stage Liver Disease; SBP, spontaneous bacterial peritonitis; UTI, urinary tract infection; WBC, white blood cell.

Source: Adapted from Bajaj et al. ${ }^{12}$ 
14 Assessment and Management of Infection in Alcoholic Hepatitis Vergis et al.

Table 2 Bacteria cultured from patients enrolled into the STOPAH study

\begin{tabular}{|c|c|c|c|}
\hline \multirow[t]{2}{*}{ Organism } & \multirow{2}{*}{$\begin{array}{l}\text { Baseline } \\
\%\end{array}$} & \multicolumn{2}{|c|}{ Incident infection } \\
\hline & & $\begin{array}{l}\text { On } \\
\text { treatment \% }\end{array}$ & $\begin{array}{l}\text { Post- } \\
\text { treatment \% }\end{array}$ \\
\hline $\begin{array}{l}\text { Gram-negative } \\
\text { bacilli }\end{array}$ & $58^{a}$ & $43^{a}$ & $68^{a}$ \\
\hline $\begin{array}{l}\text { Gram-negative } \\
\text { coccus }\end{array}$ & 0 & 2 & 0 \\
\hline $\begin{array}{l}\text { Gram-positive } \\
\text { bacilli }\end{array}$ & $5^{b}$ & $7^{b}$ & $11^{\mathrm{b}}$ \\
\hline $\begin{array}{l}\text { Gram-positive } \\
\text { coccus }\end{array}$ & 28 & 42 & 18 \\
\hline Fungus & 5 & 4 & 4 \\
\hline Mixed & 5 & 2 & 0 \\
\hline
\end{tabular}

Abbreviation: STOPAH, STeroids Or Pentoxyfilline for Alcoholic Hepatitis.

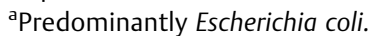

bPredominantly Clostridium difficile; equal predominance Enterococcus spp, Staphylococcus spp, Streptococcus spp.

Source: Adapted from Vergis et al. ${ }^{3}$

catheters have been sited. Additionally, chest radiography lacks specificity in distinguishing consolidated lung due to infection from sterile pulmonary fluid, both of which are common in patients with SAH. There is also uncertainty in the interpretation of fluid culture and microscopy when there is fluid leukocytosis but no microbial growth. Perhaps most importantly, the sensitivity of NACSELD criteria for detecting infection will be intimately linked to the frequency and completion of the panel of infection screening tests. Despite these limitations, the NACSELD criteria provide a reasonably objective framework for reporting definite infections for the purpose of clinical trials.

\section{Patients Not Meeting NACSELD Criteria, But Displaying Features of Systemic Inflammation}

Patients who do not meet NACSELD criteria for infection, but who exhibit features of systemic inflammation may have subclinical or occult infection. The management of these patients is uncertain; concerns over untreated infection lead to frequent prescription of antibiotic therapy, particularly in the context of the Surviving Sepsis global campaign. Markers of systemic inflammation including serum CRP are strong predictors of infection in SAH patients ${ }^{10,18}$ and elevations in CRP therefore raise suspicion for infection. Suspicion of infection should prompt urgent screening in order to make a diagnosis of definite infection. Pragmatic clinical trials demonstrate disparity between the proportion of patients diagnosed with infection and the proportion prescribed antibiotics. For example, in the STOPAH study, $12 \%$ of patients were categorized as infected at admission but $45 \%$ of patients received antibiotics. ${ }^{3}$ Similarly, a Belgian study reported that $88 \%$ of patients with SAH had received antibiotics. ${ }^{13}$

Differentiating sterile hepatic inflammation from microbial infection, which may in turn derive either from the gut or from external sources, is challenging. Liver cirrhosis can also modulate systemic inflammatory responses: hypersplenism distorts peripheral white blood cell count while $\beta$ blocking drugs prescribed for portal hypertension reduce pulse rate. Patients with cirrhosis may also have alterations in heart rate, temperature, and respiratory rate in the absence of infection as a result of a hyperdynamic circulation, hepatic encephalopathy, and tense ascites.

Almost half SAH patients fulfill two or more SIRS criteria at admission and these patients were more likely to be infected (31 vs. 10\%). ${ }^{18}$ While SIRS at admission did not predict response to corticosteroids, patients fulfilling two or more SIRS criteria at admission were more likely to develop renal dysfunction and had reduced 90-day survival (36 vs. $15 \%)$. These associations were independent of the degree of liver dysfunction and Lille score. ${ }^{18}$

In clinical practice, patients exhibiting an inflammatory response without satisfying NACSELD criteria for infection are likely to receive antibiotic therapy. To capture this subclinical infection, trials may add a further category of suspected infection, wherein patients are prescribed either new antibiotics or a change in antibiotic.

\section{Biomarkers for Infection}

Biomarkers are required for diagnosis of subclinical infection to: (1) improve antibiotic stewardship; (2) objectively describe infection in clinical trials; and as a result of more accurate antibiotic and immunosuppressive therapy; (3) prevent infection-related mortality. Biomarkers for infection seek to either define host immunological responses that are specific to infection rather than to systemic

Table 3 Comparison of top five bacterial species cultured from patients enrolled into the STOPAH and global epidemiology of infection in cirrhosis study

\begin{tabular}{|l|l|l|l|}
\hline Organism & STOPAH (UK) (\%) & Europe (\%) & Global (\%) \\
\hline Escherichia coli & $45 / 72(63)$ & $127 / 290(44)$ & $266 / 592(45)$ \\
\hline Klebsiella pneumoniae & $13 / 72(18)$ & $51 / 290(18)$ & $143 / 592(24)$ \\
\hline Staphylococcus aureus & $11 / 72(15)$ & $37 / 290(13)$ & $78 / 592(13)$ \\
\hline Enterococcus faecalis & $2 / 72(3)$ & $36 / 290(12)$ & $52 / 592(9)$ \\
\hline Enterococcus faecium & $2 / 72(3)$ & $39 / 290(13)$ & $53 / 592(9)$ \\
\hline
\end{tabular}

Abbreviation: STOPAH, STeroids or Pentoxyfilline for Alcoholic Hepatitis.

Source: Adapted from Vergis et $\mathrm{al}^{3}$ and Piano et al. ${ }^{46}$ 
inflammation; or to detect the pathogen with greater sensitivity and specificity than conventional culture either by detection of pathogen DNA or by detection of pathogensecreted proteins. Performance parameters for these candidate biomarkers are summarized in - Table 4. Similar to reported frequency of infection in SAH, performance parameters for infection biomarkers vary widely between studies.

\section{Biomarkers for Clinical Infection}

Biomarkers that improve the diagnosis of active clinical infection have been evaluated for patients with SAH. Serum CRP and procalcitonin (PCT) have been specifically studied in SAH patients with and without infection (-Table 4). Patients with SAH have higher levels of PCT and CRP even in the absence of infection. Good performance for CRP and PCT was achieved in a small cohort of patients comprising 11 patients with SIRS but no infection versus 29 patients with SIRS and infection. ${ }^{20}$ Of note, these cohorts underwent comprehensive infection screening, requiring three samples of blood and urine for culture on three consecutive days, as well as ascitic fluid, sputum culture, and chest X-ray. Samples for PCT and CRP were drawn within 24 hours of admission. ${ }^{20}$ In contrast, other retrospective cohort studies have sampled biomarkers at the time of liver biopsy ${ }^{18}$ or at the time of starting immunosuppressive medication for patients in whom any admission infection had been controlled. ${ }^{3}$ Concurrent antimicrobial therapy may have a substantial impact on performance of candidate biomarkers. In the STOPAH study, for example, there was a significant interaction between 16S bacterial DNA level and antibiotic use, and samples from patients receiving antibiotics at the time of sampling were excluded from further analysis. ${ }^{3}$ Accordingly, $\mathrm{PCT}^{21}$ did not correlate with either baseline or incident infection when measured in 708 patients from the STOPAH study in whom baseline infection had been controlled with antibiotics. 22

\section{Biomarkers for Subclinical Infection}

Biomarkers that predict incident infection have received comparatively little attention. Michelena et al found that higher levels of serum endotoxin were associated with an increased risk of developing incident infection. ${ }^{18}$ In line with this, elevated 16S ribosomal DNA from whole blood predicted infections for 265 patients treated with corticosteroid within the first week, with area under receiver operating curve (AUROC) 0.704. High endotoxin and 16S bacterial DNA levels also correlated with Lille score in predicting response to corticosteroid. ${ }^{3,18}$ Elevated bacterial DNA levels before therapy predicted a greater than fourfold risk of developing infection after initiation of corticosteroid therapy. Whether 165 ribosomal DNA is able to guide antibiotic and/or prednisolone prescription in SAH patients is unknown and should be the subject of future work. PCT, CRP, and WCC have been assessed in a small study of 42 intensively screened SAH patients and demonstrated AUROCs of $0.73,0.75$, and 0.72 to predict the development of infection within the subsequent 2 weeks. ${ }^{23}$

Improvements to nucleic acid-based detection of pathogen are expected. Conventional quantitative polymerase chain reaction uses relative estimations against reference standards or endogenous controls. In contrast, digital PCR will allow absolute quantification of target sequences to the level of a single copy, improving sensitivity as well is increasing multiplexing capabilities. ${ }^{24}$

There is emerging use of multiplex quantitative PCR in clinical laboratories, but at present, the utility is in rapidly identifying bacteria in culture media that has already shown substantial bacterial growth, rather than for the identification of low levels of bacteremia representing subclinical infection that may activate in the setting of immunosuppression. ${ }^{25}$

PCR-based techniques are inherently slow and expensive; they require lengthy DNA extraction protocols before utilization of expensive thermocycler equipment. The utility of

Table 4 Performance of infection biomarkers in SAH

\begin{tabular}{|c|c|c|c|}
\hline Biomarker & Pathogen & Performance for clinical infection & Performance for subclinical infection \\
\hline Serum CRP & $\begin{array}{l}\text { Bacteria, virus, } \\
\text { fungi }\end{array}$ & $\begin{array}{l}\text { Kumar et } \mathrm{al}^{13}: \text { AUROC } 0.81 \\
\text { Michelena et } \mathrm{al}^{3}: \text { ND } \\
\text { Louvet et } \mathrm{al}^{6}: \text { independent predictor }\end{array}$ & Michelena et $\mathrm{al}^{3}:$ ND \\
\hline Serum PCT & Bacteria & $\begin{array}{l}\text { Kumar et al }{ }^{13}: \text { AUROC } 0.83 \\
\text { Michelena et } \mathrm{al}^{3}: \\
\text { NPV/PPV } 71 / 83 \% \\
\text { Atkinson et } \mathrm{al}^{14}: \text { ND }\end{array}$ & $\begin{array}{l}\text { Atkinson et } \mathrm{al}^{14}: \text { ND } \\
\text { Michelena et } \mathrm{al}^{3}: \text { ND }\end{array}$ \\
\hline White cell count & $\begin{array}{l}\text { Bacteria, virus, } \\
\text { fungi }\end{array}$ & Louvet et $\mathrm{al}^{6}: \mathrm{ND}$ & Vergis and Atkinson ${ }^{8}:$ ND \\
\hline Serum lipopolysaccharide & Bacteria & Michelena et $\mathrm{al}^{3}:$ ND & $\begin{array}{l}\text { Michelena et } \text { al }^{3}: 59 \% \text { vs } 29 \% \\
\text { high vs low LPS }(p=0.03)\end{array}$ \\
\hline $\begin{array}{l}\text { Whole blood } 165 \\
\text { ribosomal DNA }\end{array}$ & Bacteria & Vergis and Atkinson ${ }^{8}:$ ND & Vergis and Atkinson ${ }^{8}$ : AUROC $0.70^{\text {a }}$ \\
\hline$\beta$-d-glucan & Fungi & - & - \\
\hline Galactomannan & Fungi & - & - \\
\hline
\end{tabular}

Abbreviations: AUROC, area under receiver operating curve; CRP, C-reactive protein; DNA, deoxyribonucleic acid; LPS, ND, nondiscriminatory; NPV, negative predictive value; PCT, procalcitonin; PPV, positive predictive value; SAH, severe alcoholic hepatitis.

${ }^{\mathrm{a}}$ For patients treated with prednisolone. 
PCR in providing a rapid guide for clinicians to make therapeutic decisions is therefore limited. Novel isothermal amplification techniques can achieve similar specificity and sensitivity, without the need for DNA extraction ${ }^{26}$ or expensive thermocycling, and were used in the field during the recent Ebola epidemic. ${ }^{27}$ Speed and low cost make isothermal nucleic acid amplification techniques attractive.

\section{Biomarkers for Fungal Infection}

Fungal infection is even less likely than bacterial infection to yield positive microbial culture. ${ }^{28}$ As a result, available data for fungal infection are likely to underestimate prevalence. In a Belgian cohort of SAH patients with a high rate of admission to intensive care, intensive screening for invasive aspergillosis yielded infection rates of 15/96 (16\%). All of these patients died, and invasive aspergillosis was an independent predictor of death. ${ }^{13}$

Currently available biomarkers for fungal infection center around two components of fungal cell wall: $\beta$-d-glucan and galactomannan (GM). Neither assay has been validated in the setting of SAH. Most studies evaluating performance have been in patients with hematological malignancy. GM is detected by enzyme immunoassay. $\beta$-d-glucan is measured by the limulus amebocyte lysate (LAL) assay after removal of clotting factor $\mathrm{C}$ from the limulus clotting cascade: this prevents activation of the cascade to lipopolysaccharide (LPS) that occurs when LAL is used to measure endotoxin. $^{29}$

A broad range of sensitivity and specificity values for these assays are found in published literature, from 38 to $100 \%$ and 45 to $99 \%$, respectively, with similar ranges observed for the positive predictive value (PPV: $30-89 \%$ ) and negative predictive value (73-97\%). ${ }^{30-34}$ This heterogeneity reflects the broad range of clinical scenarios in which these assays have been tested. These tests appear to have the best performance in patients with hematological malignancies who have undergone stem cell transplantation and who have frequent antigen testing. However, sensitivity and PPV for $\beta$-d-glucan are low even when serial testing is used, such that a negative $\beta$-d-glucan result cannot be used to exclude the possibility of invasive fungal disease. ${ }^{35}$ Similarly in a meta-analysis of 27 studies, GM had a pooled sensitivity of $61 \%$ for patients with proven or probable invasive aspergillosis. ${ }^{36}$ The clinical utility of these tests at present is therefore restricted to patients who have hematological disease and neutropenia presenting with symptoms of invasive fungal infection and who test repeatedly positive for $\beta$-d-glucan or GM.

\section{Interaction with Host Immunity}

Host immunity can now be described by phenotypic and functional biomarkers that are readily measured by flow or mass cytometry. A raft of data points to specific immune defects which contribute to increased susceptibility of infection in hospitalized patients. The ExPRES-sepsis cohort study linked monocyte human leucocyte antigen-DR isotype (HLA$\mathrm{DR}$ ) and neutrophil programmed death (PD)-1 expression to the development of sepsis. ${ }^{37}$

\section{Baseline Immune Defects}

Infection in patients with ACLF is associated with expansion of myeloid- derived suppressor cells, while PD-1 expression on $\mathrm{T}$ cells in $\mathrm{SAH}$ is linked to reduced interferon (IFN)- $\gamma$ secretion and increased interleukin (IL)10 production as well as impaired neutrophil antimicrobial activities. ${ }^{38}$ Aberrant neutrophil phagocytosis, ${ }^{39}$ monocyte oxidative burst, ${ }^{23}$ and mucosa-associated invariant $\mathrm{T}$-cell functions ${ }^{40}$ have been linked to the development of infection in SAH.

The thresholds at which subclinical bacteremia translate into clinical infection for patients with differing degrees of immune paresis has not been defined. Understanding interactions between host immune responses and circulating pathogen load is likely to unlock precision therapy for SAH patients with infection.

\section{Incident (Treatment Related) Immune Defects}

Few data describe the natural history of immune defects in SAH but the effect of therapy on circulating immunity has been explored. Oxidative burst was not affected by three- or sevendays' prednisolone therapy in neutrophils or monocytes, respectively. ${ }^{23,41}$ However, the combination of 3 days' $10 \mathrm{mg} / \mathrm{kg}$ infliximab (IFX) and prednisolone $40 \mathrm{mg}$ daily reduced neutrophil oxidative burst and corresponded to an increased rate of serious infections. ${ }^{41}$ Proinflammatory cytokine production (IL-8), measured ex vivo, was also significantly lower in the IFX-treated group. ${ }^{41}$ Binding of tumour necrosis factor (TNF)- $\alpha$ with etanercept ${ }^{42}$ similarly resulted in higher rates of serious infection. Conversely, a decreased rate of infection was noted when patients were treated with 5 days intravenous $N$-acetylcysteine alongside prednisolone. ${ }^{43}$

\section{Management of Infection for Patients with Severe Alcoholic Hepatitis}

\section{Selection of Antimicrobial Therapy}

There is no evidence that pathogens or their drug-resistance patterns for patients with a diagnosis of SAH differ from that of patients suffering the clinical syndrome of acute decompensation of cirrhosis (AD). Guidelines and data from the field of decompensated cirrhosis therefore remain relevant. A comparison of pathogens cultured from recent large clinical studies for patients with SAH and AD worldwide is given in - Table 2.

Two empirical antibiotic strategies are described for patients with AD: classical strategies involve first-, second-, or third-generation cephalosporins, amoxicillin-clavulanic acid, or quinolones. In contrast, MDR strategies include piperacillin-tazobactam, carbapenems, or ceftazidime/cefepime \pm glycopeptides or linezolid/daptomycin. ${ }^{44}$ Further guidance is offered by a position statement from the European Association for Study of the Liver ${ }^{45}$ : community acquired infection, namely infections that develop within the first 48 hours of hospital admission, should be treated with classical antibiotic strategies while nosocomial infection, developing after the first 48 hours of hospital admission, requires antibiotics that cover MDR organisms. Antibiotics used in the STOPAH study are compared with those used in AD globally in - Table 6. While 
Table 5 Comparison of the top five sites of infection in patients enrolled into the STOPAH and Global Epidemiology of Infection in Cirrhosis Study

\begin{tabular}{|l|l|l|l|}
\hline Site & STOPAH (UK) & Europe $^{48}$ & Global $^{48}$ \\
\hline SPB & $76 / 418(18)$ & $111 / 655(17)$ & $354 / 1302(27)$ \\
\hline Urinary & $65 / 418(16)$ & $143 / 655(22)$ & $289 / 1302(22)$ \\
\hline Lung & $192 / 418(46)$ & $90 / 655(14)$ & $242 / 1302(19)$ \\
\hline SSTI & $39 / 418(9)$ & $41 / 655(6)$ & $101 / 1302(8)$ \\
\hline Bacteremia & $46 / 418(11)$ & $49 / 655(7)$ & $100 / 1302(8)$ \\
\hline
\end{tabular}

Abbreviations: SPB, spontaneous bacterial peritonitis; SSTI, skin and soft tissue infection; STOPAH, STeroids or Pentoxyfilline for Alcoholic Hepatitis.

Source: Adapted from Vergis et al ${ }^{3}$ and Piano et al. ${ }^{46}$

there appears to less frequent use of third-generation cephalosporins in $\mathrm{AH}$ compared with $\mathrm{AD}$, these are likely to reflect differences in regional antibiotic policy between the UK and international centers ( $\mathbf{- T a b l e ~} \mathbf{6}$ ).

\section{Infection with Multidrug Resistant Organisms}

Recent data point to a substantial and rising proportion of infections involving MDR organisms in patients with liver cirrhosis. In 6 years from 2011 to 2017, the prevalence of MDR organisms found in positive cultures from patients with cirrhosis in Europe rose from 30 to $38 \%{ }^{44}$ These data align with a study of the global epidemiology of bacterial infection in cirrhosis, reporting global prevalence of MDR infection to be $34 \%$, with higher prevalence in Asia (50\%). ${ }^{46}$

Independent risk factors for the development of MDR infection were antibiotics within 3 months of hospitalization; prior health care exposure; and site of infection, with pneumonia and urinary infection being at particularly high risk. ${ }^{46}$ Nosocomial infection had an independent odds ratio (OR) of 2.74 for MDR infection, while intensive care unit admission was also relevant (OR: 2.09). These MDR infections were associated with a higher incidence of septic shock and higher in hospital mortality in patients with AD. Previous studies in patients with SAH demonstrate the negative impact of nosocomial infections on outcome, ${ }^{3,10}$ while studies involving patients with AD and ACLF confirm that second infections portend the worst prognosis. ${ }^{47}$ Importantly, if the first-line antibiotic was inadequate, there was a fourfold higher risk of death at 28 days, ${ }^{44}$ likely related to the delay in delivering the effective antibiotic. These data question current approaches to antibiotic therapy, namely the use of classical antibiotic strategies for all patients with community acquired infection first line. ${ }^{45} \mathrm{~A}$ personalized approach, taking into account a patient's recent antimicrobial and healthcare history in the selection of the first-line antibiotic, may lead to the right antibiotic being prescribed first time and improved patient outcomes.

Management of SAH fundamentally differs from AD in the use of immunosuppressive medication for treatment of hepatic inflammation. Post-hoc analysis of infection data from the STOPAH study clearly demonstrated that for patients with baseline infection there was a clear survival benefit in continuing antibiotic therapy concurrently with prednisolone. ${ }^{3}$

\section{Antibiotic Prophylaxis}

The importance and frequency of infection in patients with $\mathrm{SAH}$ have increased interest in antibiotic prophylaxis. Antibiotic prophylaxis may improve prognosis in two ways. First, it may reduce the risk of bacteremia progressing to sepsis. Second, prophylaxis may reduce bacterial load in the gastrointestinal tract and reduce the translocation of pathogens that are able to cause infection.

Whether there is survival benefit in the prescription of antibiotic prophylaxis to all patients receiving corticosteroid immunosuppression is currently unclear pending the reports from active clinical trials. In the Antibiocor study

Table 6 Comparison of antibiotic therapy prescribed in the STOPAH and in Global Epidemiology of Infection in Cirrhosis by Class

\begin{tabular}{|l|l|l|}
\hline Antibiotic & $\begin{array}{l}\text { Severe alcoholic hepatitis (UK) \% } \\
\text { of total antibiotic prescriptions }\end{array}$ & $\begin{array}{l}\text { Acute decompensation of } \\
\text { cirrhosis }\left(\mathbf{g l o b a l}^{\mathrm{a}}\right) \% \text { of total } \\
\text { antibiotic prescriptions }\end{array}$ \\
\hline Quinolone & $133 / 2185(6)$ & $180 / 2077(9)$ \\
\hline Third-generation cephalosporin & $36 / 2185(2)$ & $523 / 2077(25)$ \\
\hline $\begin{array}{l}\text { Classic b-lactams with b-lactamase inhibitors } \\
\text { (amoxicillin-clavulanic acid) }\end{array}$ & $233 / 2185(11)$ & $365 / 2077(18)$ \\
\hline Piperacillin/tazobactam & $479 / 2185(22)$ & $288 / 2077(14)$ \\
\hline Carbapenems & $82 / 2185(4)$ & $204 / 2077(10)$ \\
\hline Glycopeptide (teicoplanin, vancomycin) & $106 / 2185(5)$ & $180 / 2077(13)$ \\
\hline Antifungal & $155 / 2185(7)$ & $45 / 2077(2)$ \\
\hline $\begin{array}{l}\text { Other (b-lactams, colistin, aminoglycosides, } \\
\text { macrolides, tetracyclines) }\end{array}$ & $671 / 2185(30)$ & $244 / 2077(12)$ \\
\hline Unspecified & $290 / 2185(13)$ & $0 / 2077(0)$ \\
\hline
\end{tabular}

Abbreviation: STOPAH, STeroids Or Pentoxyfilline for Alcoholic Hepatitis.

${ }^{a}$ Data from 46 centers including 15 from Asia, 15 from Europe, 11 from South America, 5 from North America.

Source: Adapted from Vergis et $\mathrm{al}^{3}$ and Piano et al. ${ }^{46}$ 
Table 7 Key recommendations for assessment and management of infection in patients with severe alcoholic hepatitis

1. Screen for infection with chest X-ray, blood, urine and ascitic cultures on admission and whenever there is a clinical suspicion for infection.

2. Avoid invasive catheterization whenever possible to reduce the risk of opportunistic infection.

3. If corticosteroid therapy is initiated, antibiotic therapy for prior infection should be continued alongside corticosteroid.

4. Stratify infected patients for risk of multidrug resistant infection considering the site of infection and noting recent exposure to antibiotics and healthcare environments.

(NCT02281929), antibiotic prophylaxis with co-amoxiclav for patients treated with prednisolone for SAH is tested in a randomized multicenter study to see whether it can increase survival for patients at 2 months. Secondary mortality endpoints at 3 and 6 months may reveal the impact of antibiotic prophylaxis on antimicrobial resistance in this population, which is from a region with a reported $35 \%$ prevalence of MDR organisms. ${ }^{44}$

\section{Indwelling Catheters}

Given the high susceptibility to infection, there is reluctance to use indwelling catheters for these patients. SAH patients have been recruited to studies of intravenous nutrition employing intravenous catheters. ${ }^{48-50}$ Although some benefits in reducing bilirubin and improving nitrogen balance were reported for these patients, there were no survival benefits and higher rates of infection (75 versus 33\%) in patients treated with intravenous nutrition. ${ }^{51}$ On the basis of these studies within SAH and other studies in critical care, ${ }^{52}$ use of indwelling catheters should be minimized.

Key recommendations for assessment and management of infection in patients with SAH are given in - Table 7.

\section{Conclusions and Future Directions}

Despite the substantial impact on prognosis, assessment of infection in patients with SAH is imperfect. There is considerable heterogeneity between clinical studies, which has resulted in variability in performance parameters for relevant infection biomarkers in SAH. Heterogeneity is seen in definitions of infection, antibiotic treatment of patients who do and do not meet criteria for infection, and timing of blood sampling with respect to the administration of antibiotics.

Clinical criteria for diagnosing infection in this setting have recently been standardized by the NACSELD consortium and should be used in reporting clinical trials. Prospective cohort studies using these standardized criteria for infection are required, with samples for candidate biomarker analysis ideally taken before antibiotics are administered. Technology such as digital PCR and multiparametric flow cytometry will allow the sensitive and specific evaluation of host and pathogen components of infection, while functional scores should incorporate their interaction and allow for better use of antibiotic and immunosuppressive medication.

Accurate diagnosis of infection is also essential for antibiotic stewardship programs in the face of increasing prevalence of MDR organisms. Recent medical history, site of infection, and local resistance patterns should help inform decisions on empirical antibiotic strategies. Where a high risk of MDR infection is identified, MDR covering antibiotic strategies should be instituted promptly to avoid the high mortality associated with ineffective antibiotic regimens.

Conflicts of Interest

Dr. Thursz reports personal fees from Affimune, grants from Novartis, grants from GSK, during the conduct of the study.

\section{References}

1 Lucey MR, Mathurin P, Morgan TR. Alcoholic hepatitis. N Engl J Med 2009;360(26):2758-2769

2 Thursz MR, Richardson P, Allison M, et al; STOPAH Trial. Prednisolone or pentoxifylline for alcoholic hepatitis. N Engl J Med 2015; 372(17):1619-1628

3 Vergis N, Atkinson SR, Knapp S, et al. In patients with severe alcoholic hepatitis, prednisolone increases susceptibility to infection and infection-related mortality, and is associated with high circulating levels of bacterial DNA. Gastroenterology 2017;152 (05):1068-1077.e4

4 Dellinger RP, Carlet JM, Masur H, et al. Surviving sepsis campaign guidelines for management of severe sepsis and septic shock. Crit Care Med 2004;32(03):858-873

5 Dellinger RP, Levy MM, Carlet JM, et al. Surviving sepsis campaign: international guidelines for management of severe sepsis and septic shock: 2008. Intensive Care Med 2008;34(01):17-60

6 Rhodes A, Evans LE, Alhazzani W, et al. Surviving sepsis campaign: International Guidelines for Management of Sepsis and Septic Shock: 2016. Intensive Care Med 2017;43(03):304-377

7 McPhail MJ, Shawcross DL, Abeles RD, et al. Increased survival for patients with cirrhosis and organ failure in liver intensive care and validation of the chronic liver failure-sequential organ failure scoring system. Clin Gastroenterol Hepatol 2015;13(07):1353-1360.e8

8 Schmidt ML, Barritt AS, Orman ES, Hayashi PH. Decreasing mortality among patients hospitalized with cirrhosis in the United States from 2002 through 2010. Gastroenterology 2015; 148(05):967-977.e2

9 Karakike E, Moreno C, Gustot T. Infections in severe alcoholic hepatitis. Ann Gastroenterol 2017;30(02):152-160

10 Louvet A, Wartel F, Castel H, et al. Infection in patients with severe alcoholic hepatitis treated with steroids: early response to therapy is the key factor. Gastroenterology 2009;137(02):541-548

11 Arroyo V, Moreau R, Kamath PS, et al. Acute-on-chronic liver failure in cirrhosis. Nat Rev Dis Prim 2016;2:16041

12 Cabré E, Rodríguez-Iglesias P, Caballería J, et al. Short- and longterm outcome of severe alcohol-induced hepatitis treated with steroids or enteral nutrition: a multicenter randomized trial. Hepatology 2000;32(01):36-42

13 Gustot T, Maillart E, Bocci M, et al. Invasive aspergillosis in patients with severe alcoholic hepatitis. J Hepatol 2014;60(02):267-274

14 Forrest E, Storey N, Rohit S, et al. Baseline neutrophil to lymphocyte ratio can identify favourable corticosteroid response in alcoholic hepatitis. J Hepatol 2017;66(01):S99

15 Forrest EH, Atkinson SR, Richardson P, et al; STOPAH trial Management Group. Application of prognostic scores in the STOPAH trial: discriminant function is no longer the optimal scoring system in alcoholic hepatitis. J Hepatol 2018;68(03):511-518 
16 Sersté T, Moreno C, Gustot T. Reply to: "Prevalent acute-onchronic liver failure and response to corticosteroids in alcoholic hepatitis". J Hepatol 2018;69(05):1201-1202

17 Sersté T, Cornillie A, Njimi H, et al. The prognostic value of acuteon-chronic liver failure during the course of severe alcoholic hepatitis. J Hepatol 2018;69(02):318-324

18 Michelena J, Altamirano J, Abraldes JG, et al. Systemic inflammatory response and serum lipopolysaccharide levels predict multiple organ failure and death in alcoholic hepatitis. Hepatology 2015;62(03):762-772

19 Bennett JE, Dolin R, Blaser MJ. Mandell, Douglas, and Bennett's Principles and Practice of Infectious Diseases. Vol. 1-2. Amsterdam, The Netherlands: Elsevier; 2004

20 Kumar K, Mohindra S, Raj M, Choudhuri G. Procalcitonin as a marker of sepsis in alcoholic hepatitis. Hepatol Int 2014;8(03):436-442

21 Reinhart K, Meisner M. Biomarkers in the critically ill patient: procalcitonin. Crit Care Clin 2011;27(02):253-263

22 Atkinson SR, Maurice J, Vergis N, Forrest E, Thursz MR. Serum procalcitonin correlates with baseline renal function and predicts mortality in severe alcoholic hepatitis. J Hepatol 2017;66(01):S117

23 Vergis N, Khamri W, Beale K, et al. Defective monocyte oxidative burst predicts infection in alcoholic hepatitis and is associated with reduced expression of NADPH oxidase. Gut 2017;66(03):519-529

$24 \mathrm{Ou} \mathrm{C-Y,} \mathrm{Vu} \mathrm{T,} \mathrm{Grunwald} \mathrm{JT,} \mathrm{et} \mathrm{al.} \mathrm{An} \mathrm{ultrasensitive} \mathrm{test} \mathrm{for} \mathrm{profiling}$ circulating tumor DNA using integrated comprehensive droplet digital detection. Lab Chip 2019;19(06):993-1005

25 MacVane SH, Nolte FS. Benefits of adding a rapid PCR-based blood culture identification panel to an established antimicrobial stewardship program. J Clin Microbiol 2016;54(10):2455-2463

26 Rosenstierne MW, Jensen CE, Fomsgaard A. Rapid, safe, and simple manual bedside nucleic acid extraction for the detection of virus in whole blood samples. J Vis Exp 2018 Doi: 10.3791/ 58001. (epub ahead of print)

27 Magro L, Jacquelin B, Escadafal C, et al. Paper-based RNA detection and multiplexed analysis for Ebola virus diagnostics. Sci Rep 2017;7(01):1347

28 Cuenca-Estrella M, Verweij PE, Arendrup MC, et al; ESCMID Fungal Infection Study Group. ESCMID* guideline for the diagnosis and management of Candida diseases 2012: diagnostic procedures. Clin Microbiol Infect 2012;18(Suppl 7):9-18

29 Iwanaga S. Biochemical principle of Limulus test for detecting bacterial endotoxins. Proc Jpn Acad, Ser B, Phys Biol Sci 2007;83 (04):110-119

30 Sahin U, Toprak SK, Atilla PA, Atilla E, Demirer T. An overview of infectious complications after allogeneic hematopoietic stem cell transplantation. J Infect Chemother 2016;22(08):505-514

31 Marchetti O, Lamoth F, Mikulska M, Viscoli C, Verweij P, Bretagne $S$; European Conference on Infections in Leukemia (ECIL) Laboratory Working Groups. ECIL recommendations for the use of biological markers for the diagnosis of invasive fungal diseases in leukemic patients and hematopoietic SCT recipients. Bone Marrow Transplant 2012;47(06):846-854

32 Karageorgopoulos DE, Vouloumanou EK, Ntziora F, Michalopoulos A, Rafailidis PI, Falagas ME. $\beta$-D-glucan assay for the diagnosis of invasive fungal infections: a meta-analysis. Clin Infect Dis 2011; 52(06):750-770

33 Senn L, Robinson JO, Schmidt S, et al. 1,3-Beta-D-glucan antigenemia for early diagnosis of invasive fungal infections in neutropenic patients with acute leukemia. Clin Infect Dis 2008; 46(06):878-885

34 Ellis M, Al-Ramadi B, Finkelman M, et al. Assessment of the clinical utility of serial $\beta$-D-glucan concentrations in patients with persistent neutropenic fever. J Med Microbiol 2008;57(Pt 3):287-295

35 Lamoth F, Cruciani M, Mengoli C, et al. $\beta$-glucan antigenemia assay for the diagnosis of invasive fungal infections in patients with hematological malignancies: A systematic review and metaanalysis of cohort studies from the third European Conference on Infections in Leukemia (ECIL-3). Clin Infect Dis 2012;54(05): 633-643

36 Pfeiffer CD, Fine JP, Safdar N. Diagnosis of invasive aspergillosis using a galactomannan assay: a meta-analysis. Clin Infect Dis 2006;42(10):1417-1427

37 Shankar-Hari M, Datta D, Wilson J, et al. Early PREdiction of sepsis using leukocyte surface biomarkers: the ExPRES-sepsis cohort study. Intensive Care Med 2018;44(11):1836-1848

38 Markwick LJ, Riva A, Ryan JM, et al. Blockade of PD1 and TIM3 restores innate and adaptive immunity in patients with acute alcoholic hepatitis. Gastroenterology 2015;148(03):590-602.e10

39 Mookerjee RP, Stadlbauer V, Lidder S, et al. Neutrophil dysfunction in alcoholic hepatitis superimposed on cirrhosis is reversible and predicts the outcome. Hepatology 2007;46(03):831-840

40 Riva A, Patel V, Kurioka A, et al. Mucosa-associated invariant T cells link intestinal immunity with antibacterial immune defects in alcoholic liver disease. Gut 2018;67(05):918-930

41 Naveau S, Chollet-Martin S, Dharancy S, et al; Foie-Alcool group of the Association Française pour l'Etude du Foie. A double-blind randomized controlled trial of infliximab associated with prednisolone in acute alcoholic hepatitis. Hepatology 2004;39(05): 1390-1397

42 Boetticher NC, Peine CJ, Kwo P, et al. A randomized, doubleblinded, placebo-controlled multicenter trial of etanercept in the treatment of alcoholic hepatitis. Gastroenterology 2008;135 (06):1953-1960

43 Nguyen-Khac E, Thevenot T, Piquet M-A, et al; AAH-NAC Study Group. Glucocorticoids plus N-acetylcysteine in severe alcoholic hepatitis. N Engl J Med 2011;365(19):1781-1789

44 Fernández J, Prado V, Trebicka J, et al; European Foundation for the Study of Chronic Liver Failure (EF-Clif). Multidrug-resistant bacterial infections in patients with decompensated cirrhosis and with acute-on-chronic liver failure in Europe. J Hepatol 2019;70 (03):398-411

45 Jalan R, Fernandez J, Wiest R, et al. Bacterial infections in cirrhosis: a position statement based on the EASL Special Conference 2013. J Hepatol 2014;60(06):1310-1324

46 Piano S, Singh V, Caraceni P, et al. Epidemiology and effects of bacterial infections in patients with cirrhosis worldwide. Gastroenterology 2019;156(05):1368-1380

47 Bajaj JS, O'Leary JG, Reddy KR, et al; NACSELD. Second infections independently increase mortality in hospitalized patients with cirrhosis: the North American consortium for the study of endstage liver disease (NACSELD) experience. Hepatology 2012;56 (06):2328-2335

48 Bajaj JS, Heuman DM, Sanyal AJ, et al. Modulation of the metabiome by rifaximin in patients with cirrhosis and minimal hepatic encephalopathy. PLoS One 2013;8(04):e60042

49 Achord JL. A prospective randomized clinical trial of peripheral amino acid-glucose supplementation in acute alcoholic hepatitis. Am J Gastroenterol 1987;82(09):871-875

50 Bonkovsky HL, Singh RH, Jafri IH, et al. A randomized, controlled trial of treatment of alcoholic hepatitis with parenteral nutrition and oxandrolone. II. Short-term effects on nitrogen metabolism, metabolic balance, and nutrition. Am J Gastroenterol 1991;86 (09):1209-1218

51 Simon D, Galambos JT. A randomized controlled study of peripheral parenteral nutrition in moderate and severe alcoholic hepatitis. J Hepatol 1988;7(02):200-207

52 Templeton A, Schlegel M, Fleisch F, et al. Multilumen central venous catheters increase risk for catheter-related bloodstream infection: prospective surveillance study. Infection 2008;36(04): 322-327 